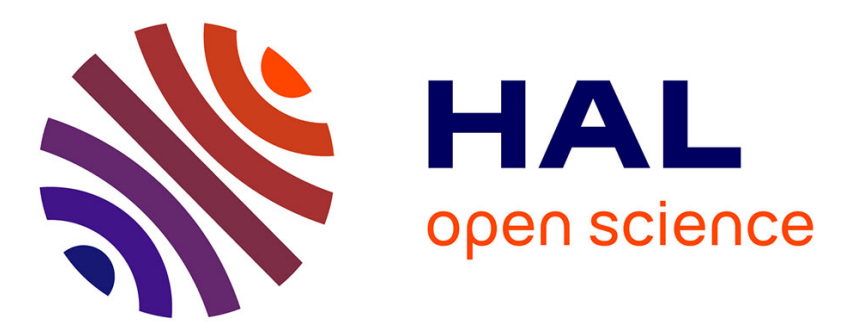

\title{
Experiments on Tracer Diffusion in Thin Free-Standing Liquid-Crystal Films
}

J Bechhoefer, J.-C Géminard, L. Bocquet, P Oswald

\section{To cite this version:}

J Bechhoefer, J.-C Géminard, L. Bocquet, P Oswald. Experiments on Tracer Diffusion in Thin Free-Standing Liquid-Crystal Films. Physical Review Letters, 1997, 10.1103/PhysRevLett.79.4922 . hal-02178729

\section{HAL Id: hal-02178729 \\ https://hal.science/hal-02178729}

Submitted on 10 Jul 2019

HAL is a multi-disciplinary open access archive for the deposit and dissemination of scientific research documents, whether they are published or not. The documents may come from teaching and research institutions in France or abroad, or from public or private research centers.
L'archive ouverte pluridisciplinaire HAL, est destinée au dépôt et à la diffusion de documents scientifiques de niveau recherche, publiés ou non, émanant des établissements d'enseignement et de recherche français ou étrangers, des laboratoires publics ou privés. 


\title{
Experiments on Tracer Diffusion in Thin Free-Standing Liquid-Crystal Films
}

\author{
J. Bechhoefer,* J.-C. Géminard, L. Bocquet, and P. Oswald \\ Laboratoire de Physique de l'E.N.S. de Lyon, 46 Allée d'Italie, 69364 Lyon Cedex
}

(Received 22 July 1997)

\begin{abstract}
Using laser-induced photobleaching of a fluorescent tracer molecule, we study diffusion in freestanding smectic films as thin as two layers. For films four or more layers thick, the in-plane diffusion constant increases in agreement with a hydrodynamic theory outlined here. Two- and three-layer films show different behavior. [S0031-9007(97)04825-4]
\end{abstract}

PACS numbers: 83.70.Jr, 68.15.+e

Stable, free-standing smectic-liquid-crystal films can be made from two to hundreds of layers thick and uniform over a $\mathrm{cm}^{2}$. They are model systems for studying the crossover between two- and three-dimensional phenomena. For many years, smectic films have been essentially used to study liquid-crystal phases and phase transitions in restricted geometry, looking at such issues as hexatic intermediate phases [1], surface freezing [2], heat capacity [3], and transition-temperature shifts [4]. More recently, physicists have realized that many other interesting and fundamental phenomena may be usefully explored in these systems, including viscosity [5], surface tension [6-8], electroconvection [9], nonlinear vibrations [10], and defects [11].

In this paper, we present the first direct study of the diffusion of a tracer molecule in free-standing films of various thicknesses. We measure how the value of the vertically averaged, in-plane diffusion constant evolves in very thin films.

Diffusion constants of small Brownian particles in a fluid can be related to microscopic mobilities via the celebrated argument of Einstein that considers the balance between impulses due to thermal fluctuations and responses determined by hydrodynamic flow [12]. (In the simplest bulk calculation, the particle is a sphere and the flow gives a Stokes force on the sphere.) Then, as we discuss below, the crossover between two- and three-dimensional diffusion is calculated by considering the (enhanced) mobility of a particle near a free surface. Because such arguments use hydrodynamics, which assumes that the tracer molecule is large compared to the surrounding fluid particles and that the system is large in all dimensions compared to the fluid particles, it is interesting to probe diffusion in situations where these assumptions are not valid. When the tracer particle is itself a molecule, the Stokes-Einstein formula still tends to be quite accurate [12]. But when the system size is confined along one or more directions, the situation is more subtle, as we shall see. Studies of micron-sized particles near walls [13] and in soap films [14] show that hydrodynamic arguments can successfully describe those cases. More recently, theoretical studies based on mode-coupling models of self diffusion near solid surfaces suggest that hydrodynamic behavior should hold down to films that are four to five molecules thick.
Here, we show that a hydrodynamical model, developed below, is valid down to $N=4$ layers thick, but that there are surprises for $N=2$ and 3 .

The liquid-crystalline compound we used was $4^{\prime}-n$ octyl-4-cyanobiphenyl (8CB) which has a smectic A mesophase for temperatures ranging from 22 to $33.4{ }^{\circ} \mathrm{C}$. A fluorescent molecular tracer, 4-(4-dihexadecylaminostyryl)- $N$-methylpyridinium iodide (DIA), is dissolved in the liquid crystal to saturation. (We use the maximal concentration of dissolved DIA molecule in order to maximize the fluorescence signal in very thin films. Measurements at lower concentration on thick films lead to the same bulk value of $D$.)

The smectic films are obtained by spreading a liquidcrystal droplet over a rigid square frame, whose area is $64 \mathrm{~mm}^{2}$. The film is kept in an oven whose temperature is stabilized to $\pm 0.1{ }^{\circ} \mathrm{C}$, to $28.4{ }^{\circ} \mathrm{C}, 5{ }^{\circ} \mathrm{C}$ below the bulk smectic-nematic transition. The film stabilizes after about half an hour: Its thickness is then homogeneous over its entire surface, and there is no fluid flow. A carefully timed and shaped current pulse in a heating wire placed $50 \mu \mathrm{m}$ below the film allows one to remove a single molecular layer [15], as discussed in detail in Ref. [11]. In this way, we could systematically reduce film thicknesses from about a hundred layers down to two, performing diffusion measurements at desired thicknesses.

The films are observed, from above, in a reflection microscope equipped with three different light sources (Fig. 1). By measuring the film's reflectivity at normal incidence as a function of wavelength, we could deduce the number of layers $N$ [8]. The measurement is unambiguous for $N<25$ and to within $2 \%$ for thicker films.

Our measurement method for the diffusion constant is an adaptation of the technique known as fluorescence recovery after photobleaching (FRAP), which has been widely used in biophysics in the study of protein diffusion in phospholipid bilayers [16]. The general principle of the measurement is that an intense laser beam is used to photobleach (destroy) the fluorescence of tracer molecules in a small region. A much weaker beam then monitors the recovery of the signal as unbleached molecules diffuse into the bleached area, leading to the recovery of the fluorescence signal. 


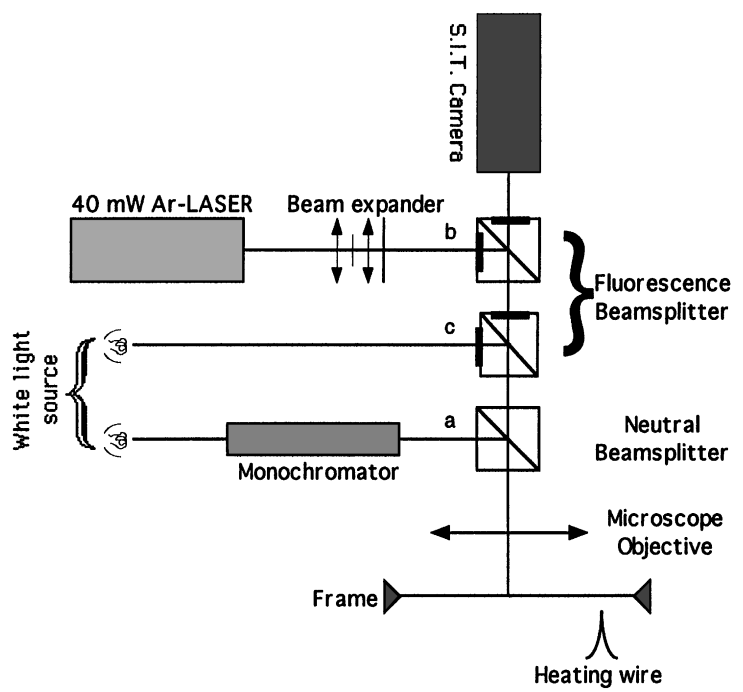

FIG. 1. Experimental setup: (a) Thickness measurement. (b) Bleaching. (c) Observation of the fluorescent light.

Our implementation of FRAP differs from the classical one of Axelrod et al. [16] in several ways. Like them, we use laser light to bleach a spot in the film. We then, however, monitor the optical image of the spot, as illuminated weakly by a halogen lamp followed by an excitation filter matched to the absorption band of the DIA dye. The fluorescence is imaged directly. There are advantages to directly imaging the bleached spot, as opposed to the classical technique of reducing the bleaching beam intensity and monitoring the recovery signal. First, the actual initial condition, which must be modeled in the former technique, may be directly observed. Second, the presence of any hydrodynamic flow is immediately apparent, while in nonimaging versions of FRAP, flow can only be inferred by its change in the time-dependence of the recovery signal. Several other groups have imaged the imprinted photobleaching pattern $[17,18]$, but our implementation differs in a number of technical details, as we will discuss in a more specialized paper. We can measure diffusion constants with a reproducibility better than $20 \%$ for the thinnest film $(N=2)$.

The bleaching of the DIA dye molecule allows one to mark the probe and to follow afterwards the time evolution of a given initial concentration profile: When a molecule absorbs a photon, it can either reemit a photon with a longer wavelength (fluorescence) or transfer its energy nonradiatively to a different molecule (often oxygen), with a given probability. The excited singlet state of oxygen is extremely reactive and can destroy the dye's fluorescence. The process is irreversible.

We bleach a spot about $R=20 \mu \mathrm{m}$ in radius by focusing the $\mathrm{TEM}_{00}$ mode of a multimode $\mathrm{Ar}^{+}$-ion laser of total power $40 \mathrm{~mW}$. The intensity profile of the beam is approximately Gaussian. Typical bleaching times are of the order of one second. (The characteristic diffusion time, $R^{2} / 4 D$, of the initial spot is about $20 \mathrm{sec}$.) The signal was weak enough to require a silicon-intensifiedtube camera (SIT). Because the absorption length of the dye is large compared to the film thickness, the local light intensity is proportional to the concentration of nonbleached molecules (Fig. 2). (We checked that there was no concentration quenching of the dye's fluorescence.) The sensitivity of the SIT camera allowed us to resolve the bleached spot over several diffusion times.

For three-dimensional diffusion in a thin film, the concentration field of nonbleached molecules $c(r, t)$ obeys a $t w o$-dimensional diffusion equation

$$
\frac{\partial c}{\partial t}=D \Delta c-\beta I_{0} c .
$$

Here, the source term $\beta I_{0} c$ accounts for the bleaching due to the observation light, whose homogeous intensity is $I_{0}$.

In Eq. (1), $D$ is a vertically averaged in-plane diffusion constant given by

$$
D=\frac{1}{N b} \int_{-N b / 2}^{+N b / 2} \rho(z) D(z) d z,
$$

where $b$ is the thickness of one layer, $z$ is the direction normal to the plane of the film, $\rho(z)$ is the steadystate concentration distribution (normalized to unity), and $D(z)$ is the local in-plane diffusion constant. Equation (2) may be proven by expanding all functions of $z$ in orthogonal polynomials [sines in the case of uniform $\rho(z)]$. Intuitively, the film is so thin compared to the lateral scale of the diffusing spot that any deviation of the concentration from the long-time steady-state field in the vertical direction relaxes quickly to zero. Note that for a smectic liquid crystal, diffusion along the molecules occurs at a different rate from diffusion in plane, so that $D$

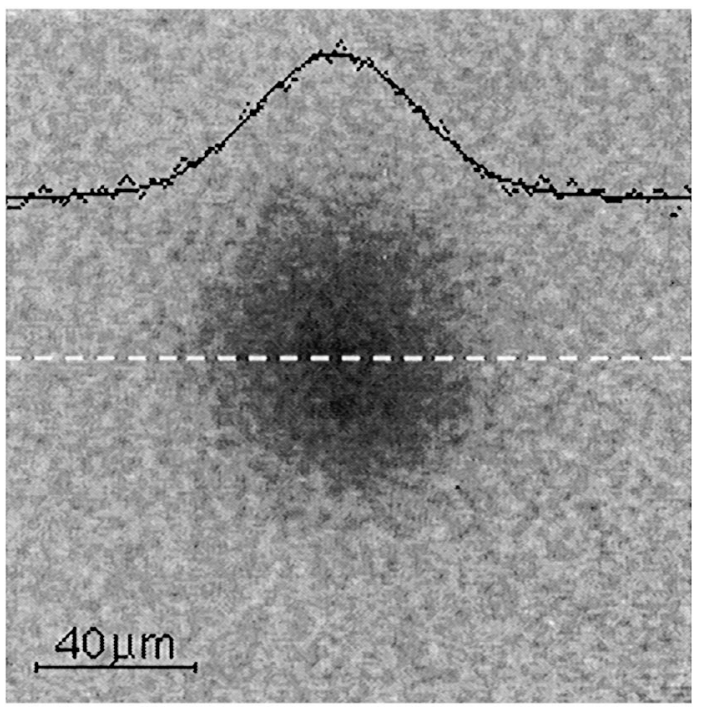

FIG. 2. Image of the film after bleaching: The black dots correspond to the concentration profile along the dashed line, the black line to the Gaussian interpolation. 
is more properly $D_{\perp}$, but Eq. (2) is also valid for diffusion in a uniaxial medium.

The long-time solution to Eq. (1) is

$$
c(r, t)=A\left(\frac{e^{-\beta I_{0} t}}{t}\right) \exp \left(-\frac{r^{2}}{4 D t}\right) .
$$

For large $t$, the bleaching of the molecules during observation affects only the amplitude of the Gaussian distribution and, thus, does not affect the spatial distribution of the bleached molecules.

The diffusion coefficient is deduced from the images by fitting a Gaussian function whose free parameters are the two coordinates of the center, the amplitude, and the width $w=\sqrt{4 D t}$. Tracking the position of the center allows us to check that there is no fluid flow in the film. The asymptotic regime is reached after about $10 \mathrm{sec}$ even if the initial profile is not strictly Gaussian and, in agreement with Eq. (3), the spot area $w^{2}$ is then proportional to $t$ (Fig. 3).

The experimental values of $D(N)$ are plotted in Fig. 4 . For $N \geq 4$, the diffusion coefficient obeys

$$
D(N)=D_{b}\left(1+\frac{N_{h}}{N}\right),
$$

where $D_{b}=(4.4 \pm 0.1) \times 10^{-8} \mathrm{~cm}^{2} \mathrm{~s}^{-1}$ is the lateral diffusion constant of the DIA in an infinitely thick film and $N_{h}=3.4 \pm 0.3$.

The $1 / N$ finite-size correction to the diffusion constant can be understood as follows: Assuming slow-enough variation of diffusion, we can write a local StokesEinstein relation between diffusion and mobility: $D(z)=$ $k_{B} T \mu(z)$. The position-dependent mobility $\mu(z)$ is evaluated by computing the force exerted on a tracer molecule when it moves at constant altitude $z$. If the film is thick enough, the contributions from the two free interfaces can be added and the mobility can be approximated by

$$
\mu(z)=\mu_{0}\left(\frac{N b}{2}+z\right)+\mu_{0}\left(\frac{N b}{2}-z\right)-\mu_{b},
$$

where $\mu_{b}=D_{b} / k_{B} T$ is the bulk mobility and $\mu_{0}(z)$ is the position-dependent mobility in a semi-infinite geometry, with a free interface located at $z=0$. For $z$ sufficiently

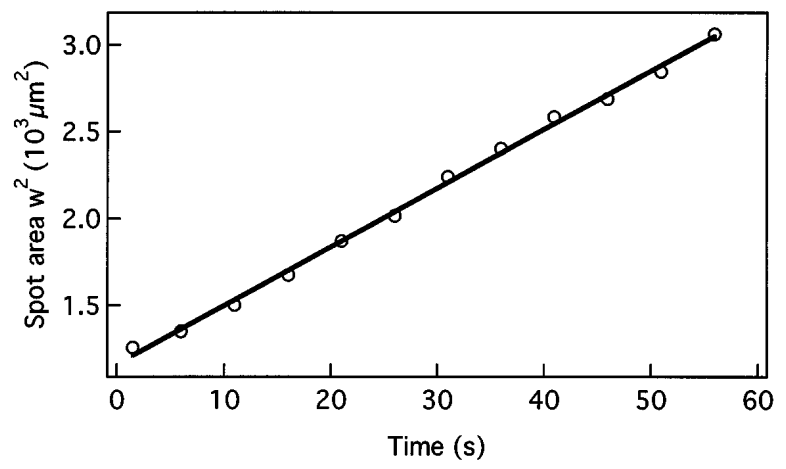

FIG. 3. Surface area versus time $(N=7)$. The linear interpolation gives $D=6.1 \times 10^{-8} \mathrm{~cm}^{2} / \mathrm{s}$. large, we obtain

$$
\mu_{0}(z) \simeq \frac{1}{\zeta_{0}[1-\phi(2 z)]} .
$$

Here, $\zeta_{0}$ is the friction coefficient on a single particle and $\phi(2 z)$ is the velocity field created by the molecule with a unit velocity on its image (located at a distance $2 z$ ) with respect to the free interface. Using the results of de Gennes [19], we have $\zeta_{0}=8 \pi \eta \sigma_{h}$, with $\eta$ the viscosity of the smectic phase and $\sigma_{h}$ the hydrodynamic radius of the tracer, and $\phi(2 z) \simeq \frac{\sigma_{h}}{2 z}+\mathcal{O}\left[\left(\frac{\sigma_{h}}{2 z}\right)^{2}\right]$. This yields, to leading order

$$
\mu_{0}(z) \simeq \mu_{b}\left(1+\frac{\sigma_{h}}{2 z}\right),
$$

with

$$
\mu_{b}=\frac{1}{\left(8 \pi \eta \sigma_{h}\right)}=\frac{D_{b}}{k_{B} T} .
$$

The presence of the free interface thus enhances the mobility of the tracer.

Next, let us denote by $\ell$ the spatial scale of the decay (in the $z$ direction) of the density profile $\rho(z)$ of the tracer away from the free surface. Using Eq. (7), Eq. (2) reduces to leading order to Eq. (4), with $N_{h}$ given by $N_{h}=\frac{2 N \sigma_{h}}{\ell} \ln \left\{\frac{N b+\ell}{N b-\ell}\right\}$. At this point, two scenarios are possible. Either the decay length $\ell$ is of the order of the film thickness $N b$, and then $N_{h} \simeq \frac{2 \sigma_{h}}{b} \ln \left\{N b / \sigma_{c}\right\}$, with $\sigma_{c}$ being a cutoff value of order $b$ (introduced in order to avoid the unphysical divergence of the mobility at the interface). Under this assumption, $N_{h}$ depends only weakly on the number of layer through the $\ln N$ term. Another possibility is that $\ell$ is smaller than the film thickness $N b$. This scenario is supported by the experimental observation that the DIA molecules crystallize out of solution when the film thickness is decreased, indicating that the tracer concentration is higher in the middle of the film than it is at the edges. This is physically plausible: since the DIA molecule is

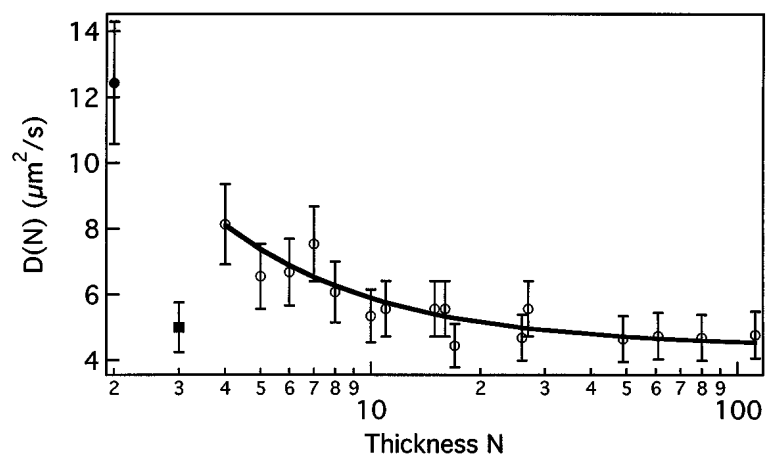

FIG. 4. Diffusion coefficient $D$ vs thickness $N$. Open circles: $N>3$; Full line: interpolation with Eq. (4). Each point corresponds to the average value of $D$ over ten measurements involving three different films. Error bars are estimated from data dispersion. 
larger than the layer thickness $b$, it behaves as if it were a small edge dislocation loop, which we know to be repelled from the free surfaces [20]. In this case, we obtain $N_{h} \simeq \frac{4 \sigma_{h}}{b}$, independent of $N$, in agreement with the experimental law. Putting numbers into the two previous estimations leads to values of $N_{h}$ of order of a few units ( $\sigma_{h}$ and $b$ being expected to be of the same order of magnitude). A semiquantitative agreement with the experimental value $N_{h}=3.4 \pm 0.3$ is thus found, which is quite reasonable in view of the qualitative nature of our theoretical description. It is, however, difficult to decide clearly between the two alternatives because of the weak $\ln N$ dependence of $N_{h}$ in the first scenario.

The theoretical prediction thus justifies the experimentally observed increase of the diffusion coefficient in free smectic films and allows one to understand the simple $1 / N$ finite-size correction. Note that a recent mode-modecoupling theory predicts that in simple fluids, the decrease in mobility near a solid surface predicted by hydrodynamic theories should also hold for a molecule as well, down to within a few (3-4) molecular thicknesses away from the wall [21].

For $N=2$ and 3 , the film thickness is smaller than the characteristic size of the diffusing particles. In this case, the hydrodynamic calculation of particle mobility near free surfaces breaks down. One still expects greater values of the diffusion coefficient, which we observe for $N=2$. On the other hand, the surprisingly small value of $D$ for three layers remains unexplained. While it is premature to speculate at length as to the reasons for the anomaly at $N=3$ (measured independently in three different films), we note that this case is distinguished in that the middle layer is adjacent to the two outer layers. One can then anticipate that there may be effects dependent on the cooperative interaction of the two surface layers. In other words, the additivity assumed in Eq. (5) is not likely to be correct, or even a good approximation.

In conclusion, the thickness of free-standing smectic films can be varied continuously from two to several hundred layers. Such films are good systems for studying the crossover between two- and three-dimensional diffusion. We studied the diffusion of a fluorescent molecule (DIA) in films of the smectic A phase $(8 \mathrm{CB}), 5^{\circ} \mathrm{C}$ below the smectic-nematic phase transition. At this temperature, the free-surface layers are liquid and the diffusion coefficient increases with decreasing thickness. The experimental results agree with a calculation based on hydrodynamics, as long as the film thickness is greater than the hydrodynamical size of the diffusing particle (about three smectic layers). On the other hand, the diffusion coefficient is surprisingly small for a three-layer film.

Further study of the $N=3$ case, as well as a systematic exploration of parameter space (dye concentra- tion and type, temperature, liquid crystal molecule) are in progress.

This work was supported by the Research Network Contract from the European Community (FMRX-CT960085) and a grant from the Centre Jacques Cartier.

* Present address: Department of Physics, Simon Fraser University, Burnaby, B.C., V5A 1S6 Canada

[1] C. C. Huang and T. Stoebe, Adv. Phys. 42, 343 (1993).

[2] B. D. Swanson et al., Phys. Rev. Lett. 62, 909 (1989).

[3] T. Pitchford, C. C. Huang, R. Pindak, and J. W. Goodby, Phys. Rev. Lett. 57, 1239 (1986); R. Geer, T. Stoebe, T. Pitchford, and C. C. Huang, Rev. Sci. Instrum. 62, 415 (1991).

[4] R. Geer, T. Stoebe, and C.C. Huang, Phys. Rev. E 48, 408 (1993).

[5] R. Pindak, D. J. Bishop, and W. O. Sprenger, Phys. Rev. Lett. 44, 1461 (1980).

[6] T. Stoebe, P Mach, and C.C. Huang, Phys. Rev. E 49, 3587 (1994).

[7] M. Eberhardt and R. B. Meyer, Rev. Sci. Instrum. 67, 2846 (1996).

[8] P. Pieranski et al., Physica (Amsterdam) 194A, 364 (1993).

[9] S. W. Morris, Liq. Cryst. Today 2, 4 (1992); S. W. Morris, J. R. de Bruyn, and A. D. May, Phys. Rev. Lett. 65, 2378 (1990).

[10] M. Brazovskaia, H. Dumoulin, and P. Pieranski, Phys. Rev. Lett. 76, 1655 (1996).

[11] J. C. Geminard, R. Holyst, and P. Oswald, Phys. Rev. Lett. 78, 1924 (1997).

[12] J. P. Hansen and I. R. McDonald, Theory of Simple Liquids (Academic Press, London, 1986).

[13] L. P. Faucheux and A. J. Libchaber, Phys. Rev. E 49, 5158 (1994).

[14] C. Cheung, Y.H. Hwang, and X.-1. Wu, Phys. Rev. Lett 76, 2531 (1996).

[15] The physics behind the layer removal is a bit subtle [12]. Briefly, because of the meniscus, the pressure in a film is lower than the ambient atmosphere. Heating locally softens the film and allows thermal fluctuations to nucleate a dislocation loop with a Burger's vector of one layer. If the loop is large enough, it will grow in size (because the air is squeezing down on it) until it reaches the film's edge (meniscus). In this way, one layer is removed.

[16] D. Axelrod, E. Koppel, J. Schlessinger, E. L. Elson, and W. W. Webb, Biophys. J. 16, 1055 (1976).

[17] B. A. Smith and H. M. McConnell, Proc. Natl. Acad. Sci. U.S.A. 75, 2759 (1978).

[18] C. Dietrich, R. Merckl, and R. Tampé, Biophys. J. 72, 1701 (1997).

[19] P. G. de Gennes, Phys. Fluids 17, 1645 (1974).

[20] L. Lejcek and P. Oswald, J. Phys. II (France) 1, 931 (1991).

[21] L. Bocquet and J. L. Barrat, Europhys. Lett. 31, 455 (1995); J. Phys. Condens. Matter 8, 9297 (1996). 